\title{
Eplerenone inhibits oxidized low-density lipoprotein- induced proliferation and migration of vascular smooth muscle cells by downregulating GPER expression
}

\author{
Dandan Wang ${ }^{1, A-D, F}$, Minglei Wang ${ }^{2, B, C, F}$, Pingping Sun ${ }^{1, C, F}$, Qiaoyan Gao $0^{3, A, E, F}$ \\ ${ }^{1}$ Nursing Department, Weihai Central Hospital, China \\ 2 Pharmacy Department, Weihai Central Hospital, China \\ ${ }^{3}$ Nursing Department, Weihai Central Hospital, China \\ A - research concept and design; $B$ - collection and/or assembly of data; $C$ - data analysis and interpretation; \\ $D$ - writing the article; $E$ - critical revision of the article; $F$ - final approval of the article
}

\section{Address for correspondence}

Qiaoyan Gao

E-mail: qiaoyang55@126.com

Funding sources

None declared

Conflict of interest

None declared

Received on July 16,2020

Reviewed on August 4, 2020

Accepted on February 19, 2021

Published online on April 29, 2021

Cite as

Wang D, Wang M, Sun P, Gao Q. Eplerenone inhibits oxidized low-density lipoprotein-induced proliferation and migration of vascular smooth muscle cells by downregulating GPER expression. Adv Clin Exp Med. 2021;30(4):405-412. doi:10.17219/acem/133484

DOI

10.17219/acem/133484

\section{Copyright}

Copyright by Author(s)

This is an article distributed under the terms of the

Creative Commons Attribution 3.0 Unported (CC BY 3.0)

(https://creativecommons.org/licenses/by/3.0/)

\begin{abstract}
Background. Excessive proliferation and migration of vascular smooth muscle cells (VSMCS) are associated with the pathogenesis of atherosclerosis (AS). Eplerenone (EPL), a novel selective aldosterone receptor blocker, plays a substantial role in the treatment of cardiovascular disease. The $G$ protein-coupled estrogen receptor (GPER) is a target of EPL as the STITCH website predicated.
\end{abstract}

Objectives. We aimed to investigate the roles of EPL in AS and identify its potential mechanisms of action.

Materials and methods. Oxidized low-density lipoprotein (ox-LDL) was employed to stimulate VSMCs to establish a cellular model of AS. The ability of cell proliferation was examined using a Cell Counting Kit-8, and the expression of proliferation-related proteins was tested using immunofluorescence staining and western blot analysis. Subsequently, cell migration and the expression of migration-associated proteins were evaluated with a wound healing assay, transwell assay and western blot analysis. Then, GPER expression was determined using western blot analysis in the absence or presence of EPL. To explore the regulatory mechanisms of EPL in ox-LDL-stimulated VSMCS, GPER was overexpressed, followed by measurement of cell proliferation and migration.

Results. The 0x-LDL stimulation notably upregulated GPER expression, whereas EPL treatment downregulated GPER expression in a dose-dependent manner. Additionally, EPL markedly inhibited proliferation and migration of VSMCs, and the highest dose of EPL resulted in the most marked effect. By contrast, GPER overexpression reversed the inhibitory effects of EPL on proliferation and migration of VSMCs.

Conclusions. Eplerenone suppressed ox-LDL-induced proliferation and migration of VSMCs partly through downregulation of GPER, providing a new mechanism of support for EPL use in the clinical treatment of AS.

Key words: atherosclerosis, proliferation, migration, vascular smooth muscle cells, eplerenone 


\section{Background}

Atherosclerosis (AS), a chronic degenerative disease of the arterial wall, is the leading cause of peripheral vascular disease and cardiac-cerebral vascular disease. ${ }^{1}$ It is well known to cause high morbidity and mortality in aged individuals worldwide. ${ }^{2,3}$ Vascular smooth muscle cells (VSMCs) are involved in the reconstruction of arterial wall by maintaining blood flow in affected vessels due to atherosclerotic alteration. ${ }^{4}$ A growing body of literature has shown that abnormal proliferation and migration of VSMCs are closely associated with AS progression. ${ }^{5,6}$ Oxidized low-density lipoprotein (ox-LDL), a well-established risk factor for AS, can induce proliferation and migration of VSMCs, thereby contributing to atherosclerotic plaque formation and progression. ${ }^{7}$ Therefore, ox-LDL was employed in the present study to stimulate VSMCs to establish an AS cell model, providing a similar environment to explore the regulatory mechanisms involved in AS.

Eplerenone (EPL), a selective aldosterone receptor antagonist, is approved by Federal Drug Administration (FDA) for the treatment of left-sided heart failure and systemic hypertension. ${ }^{8}$ Compelling evidence has indicated that EPL is effective in remedying cardiovascular diseases secondary to hypertension. ${ }^{9}$ One report supports the notion that EPL inhibits Tregs by inactivation of Kv1.3 channel to reverse cardiac fibrosis. ${ }^{10}$ Additionally, EPL can suppress proliferation of contralateral renal cells in rats with unilateral ureteral obstruction. ${ }^{11}$ What is more, EPL has been affirmed to reduce renal inflammation, interstitial cell proliferation and phenotypic changes of interstitial cells. ${ }^{12}$ The STITCH website (http:// stitch.embl.de) predicts that the $\mathrm{G}$ protein-coupled estrogen receptor (GPER), a G protein-coupled receptor coupled with Gs proteins, is a target of EPL. It has been reported that GPER promotes proliferation, invasion and migration of triple-negative breast cancer cells. ${ }^{13}$ Furthermore, blocking the function of GPER can alleviate the bisphenol A-induced proliferation of VSMCs. ${ }^{14}$ However, the role of EPL in AS and whether it functions through targeting GPER remain to be elucidated.

\section{Objectives}

In the present study, we probed the impacts of EPL on proliferation and migration of VSMCs stimulated by oxLDL, as well as its underlying molecular mechanism. This study is of great significance since it provides experimental support for EPL therapy in the clinical treatment of AS.

\section{Materials and methods}

\section{Cell culture and treatment}

Human VSMCs were purchased from China Center for Type Culture Collection (Wuhan, China) and cultured in Dulbecco's modified Eagle's medium (DMEM) containing 10\% fetal bovine serum (FBS; Gibco, Grand Island, USA) at $37^{\circ} \mathrm{C}$ in fully humidified air of $95 \%$ air and $5 \% \mathrm{CO}_{2}$. The VSMCs were seeded in six-well plates at the density of $1 \times 10^{5}$ cells/well. To construct the AS model in vitro, $100 \mu \mathrm{g} / \mathrm{mL}$ of ox-LDL (Yiyuan Biotech, Guangzhou, China) were utilized to treat cells for $48 \mathrm{~h}$. After that, the cells were treated with $0.3 \mu \mathrm{M}$, $1 \mu \mathrm{M}$ and $3 \mu \mathrm{M} \mathrm{EPL}$ (Pfizer, New York, USA) for $24 \mathrm{~h}$ to evaluate the effects of EPL on ox-LDL-induced VSMCs.

\section{Cell transfection}

Cells were plated into six-well plates $\left(1 \times 10^{6}\right.$ cells per well $)$ and transfection was performed once cells in the logarithmic growth phase reached $80 \%$ confluence. The overexpression plasmids of GPER (Oe-GPER-1 and Oe-GPER-2) and empty vector (Oe-NC) were designed and synthesized by RiboBio Co., Ltd. (Guangzhou, China). Transfection experiments were carried out with Lipofectamine 3000 (Invitrogen, Carlsbad, USA) following manufacturer's recommendations. At $24 \mathrm{~h}$ after transfection, VSMCs were collected and the successful transfection was determined using reverse transcriptionquantitative polymerase chain reaction (RT-qPCR) analysis.

\section{Cell viability assay}

A Cell Counting Kit-8 (CCK-8; Shanghai Yi Sheng Biotechnology Co. Ltd., Shanghai, China) was adopted for detecting cell viability after appropriate treatments according to standard techniques. In brief, VSMCs (about $5 \times 10^{3}$ cells/per well) were seeded in a 96-well plate. After exposure to EPL or ox-LDL, a volume of $10 \mu \mathrm{L}$ of CCK-8 solution was added to each well. Following further incubation at $37^{\circ} \mathrm{C}$ for $1 \mathrm{~h}$, the absorbance was determined at the wavelength of $450 \mathrm{~nm}$ using a microplate reader (Molecular Devices, Sunnyvale, USA).

\section{Immunofluorescence staining}

The VSMCs were plated on coverslips in 24-well plates following transfection and cultured until $80 \%$ confluence was reached. After different treatments, cells were fixed with $4 \%$ paraformaldehyde for $15 \mathrm{~min}$, followed by permeabilization with $0.05 \%$ Triton X-100 for $20 \mathrm{~min}$ at room temperature. After blocking in 5\% bovine serum albumin for $30 \mathrm{~min}$, cells were cultivated with a primary antibody against Ki67 (Cell Signaling Technology, Inc., Boston, USA) overnight at $4^{\circ} \mathrm{C}$. Following three-time washing with phosphate-buffered saline (PBS), cells were incubated DyLight ${ }^{\mathrm{TM}}$ 488-conjugated secondary antibody (Thermo Fisher Scientific, Inc., Waltham, USA) for $1 \mathrm{~h}$ at room temperature. Subsequently, the nuclei were stained with 4',6-diamidino-2-phenylindole (DAPI; Sigma-Aldrich, Merck KGaA, St. Louis, USA) for 5 min and then washed 3 times with PBS in the dark. Immunofluorescence was detected under a fluorescence microscope (Olympus Corp., Tokyo, Japan). 


\section{Wound healing assay}

The VSMCs $\left(2 \times 10^{5}\right.$ cells/well $)$ were placed in six-well plates and cultured overnight at $37^{\circ} \mathrm{C}$. Thereafter, cells were incubated with serum-free medium for $12 \mathrm{~h}$ prior to the experiment. Afterwards, a wound was gently created by a $10 \mu \mathrm{L}$ sterile pipette tip and the cell debris was washed twice with PBS. Wound closure was monitored by comparing digital photographs of the same region of interest taken at $0 \mathrm{~h}$ and $24 \mathrm{~h}$ time points using a fluorescence microscope (Olympus Corp.). Quantitative analysis of the wound healing area was performed using ImageJ software (National Institutes of Health (NIH), Bethesda, USA).

\section{Transwell migration assay}

For the transwell migration assay, serum-free media containing $5 \times 10^{4}$ VSMCs were placed into the upper chamber of a 24 -well transwell filter with $8 \mu \mathrm{m}$ pore size. The lower chamber was filled with media supplemented with $10 \%$ FBS. Migratory cells would transgress through the porous filters at $37^{\circ} \mathrm{C}$ within $24 \mathrm{~h}$. Then, VSMCs were fixed with $4 \%$ paraformaldehyde for $20 \mathrm{~min}$. Cells that migrated through the pores of the filters were stained with $1 \%$ crystal violet for $30 \mathrm{~min}$. The images were photographed under a fluorescence microscope (Olympus Corp.) and the number of migrated cells was calculated using ImageJ software.

\section{RT-qPCR analysis}

Total RNA was isolated from VSMCs using TRIzol reagent (Invitrogen) according to standard techniques. The complementary DNA (cDNA) was synthesized using a PrimeScript RT Reagent Kit (TaKaRa, Tokyo, Japan). The PCR then was performed with $2 \mu \mathrm{g}$ cDNA as the templet using Power SYBR Master Mix (Applied Biosystems, Foster City, USA) on the ABI 7500 PCR system (Applied Biosystems). The following thermocycling conditions were used: initial denaturation at $95^{\circ} \mathrm{C}$ for $7 \mathrm{~min} ; 40$ cycles of $95^{\circ} \mathrm{C}$ for $15 \mathrm{~s}$ and $60^{\circ} \mathrm{C}$ for $30 \mathrm{~s}$; and a final extension at $72^{\circ} \mathrm{C}$ for $30 \mathrm{~s}$. Sequences of the gene-specific primers were synthesized by Ribobio Co., Ltd. (Guangzhou, China). Relative expression was calculated using the $2^{-\Delta \Delta \mathrm{Ct}}$ method. Gene expression was normalized to that of glyceraldehyde 3-phosphate dehydrogenase (GAPDH).

\section{Western blot analysis}

Whole proteins were extracted using a protein lysis buffer in the presence of a protease inhibitor cocktail (Beyotime, Shanghai, China), which were further quantified using a bicinchoninic acid protein assay kit (Beyotime). Then, equal amount of total protein $(50 \mu \mathrm{g})$ was subjected to $10 \%$ SDS-PAGE electrophoresis and transferred onto polyvinylidene fluoride (PVDF) membranes (Millipore,
Billerica, USA). The membranes were subsequently blocked with $5 \%$ non-fat milk for $1 \mathrm{~h}$ at room temperature and incubated with primary antibodies (Santa Cruz Biotechnology, Dallas, USA) overnight at $4^{\circ} \mathrm{C}$. Following this, the membranes were further probed with horseradish peroxidase (HRP)-conjugated secondary antibody (Cell Signaling Technology) for $1.5 \mathrm{~h}$ at room temperature. The immunoreactive protein bands on the membranes were visualized using the Odyssey Infrared Imaging System (LI-COR Biosciences, Lincoln, USA). ImageJ software was used for quantitative analysis of the gray values of protein bands. The relative expression was normalized to the internal control GAPDH.

\section{Statistical analyses}

All data are represented as mean values \pm standard deviation (SD) from at least 3 independent experiments. Statistical analysis was conducted with GraphPad Prism v. 6 (GraphPad Software, Inc., San Diego, USA). Student's t-test was utilized to analyze data between 2 groups. Comparisons involving multiple samples were conducted using oneway analysis of variance (ANOVA) followed by Tukey's post hoc test. The acceptable value of significance was $\mathrm{p}<0.05$.

\section{Results}

\section{EPL dose-dependently inhibited cell proliferation in ox-LDL-stimulated VSMCs}

Firstly, a CCK- 8 assay was used to assess cell viability after VSMCs were treated with increasing EPL. As displayed in Fig. 1A, there was no significant effect on cell viability in response to EPL treatment $(0.3 \mu \mathrm{M}, 1 \mu \mathrm{M}$ and $3 \mu \mathrm{M})$ relative to the control group. By contrast, ox-LDL challenge significantly enhanced cell viability, which was reduced with EPL co-treatment in a dose-dependent manner (Fig. 1B). Additionally, notably elevated Ki67 expression was observed in ox-LDL-stimulated VSMCs relative to the untreated group, whereas EPL intervention significantly decreased Ki67 expression, especially under co-treatment with $3 \mu \mathrm{M}$ EPL (Fig. 1C). Consistently, EPL markedly downregulated the expression of minichromosome maintenance-2 (MCM-2) and proliferating cell nuclear antigen (PCNA), which are key proliferation-related proteins, in ox-LDL-exposed VSMCs (Fig. 1D). These results implicate that EPL attenuates proliferation of VSMCs stimulated by ox-LDL.

\section{EPL treatment suppressed migration of VSMCs induced by ox-LDL}

The influence of EPL on cell migration in ox-LDL-induced VSMCs was explored in the following experiments. Results presented in Fig. 2A,B show that ox-LDL challenge 
A

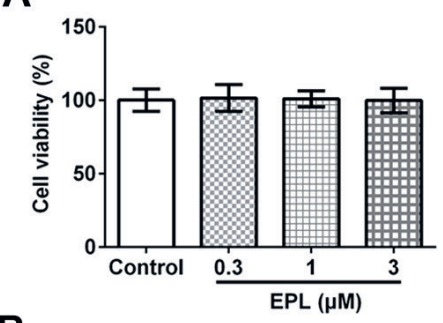

B

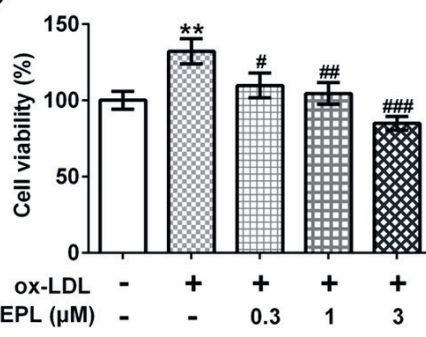

C
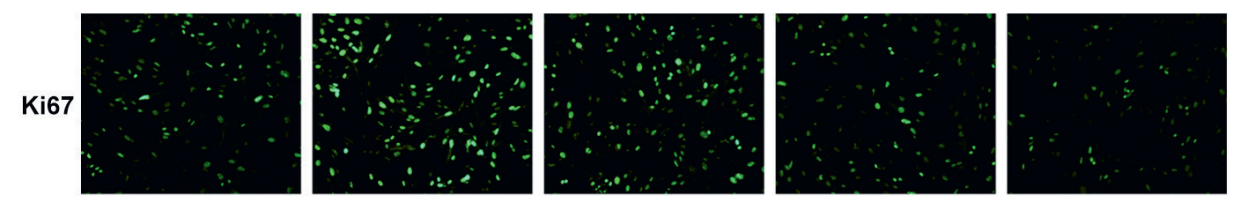

DAPI
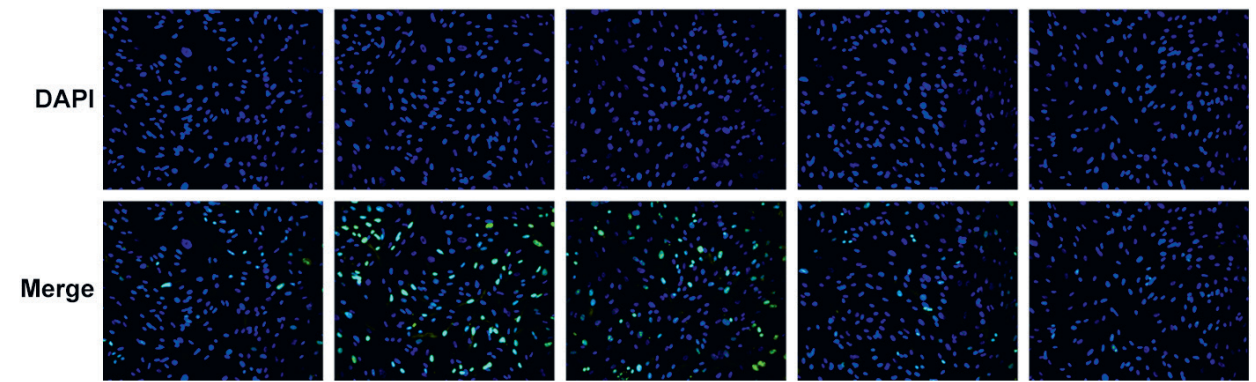

ox-LDL

EPL $(\mu M)$

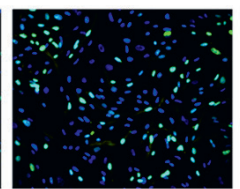

$+$

$-$

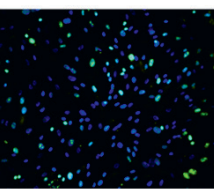

$+$

0.3

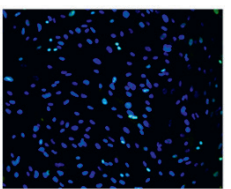

$+$

1

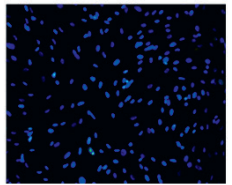

$+$

3

D
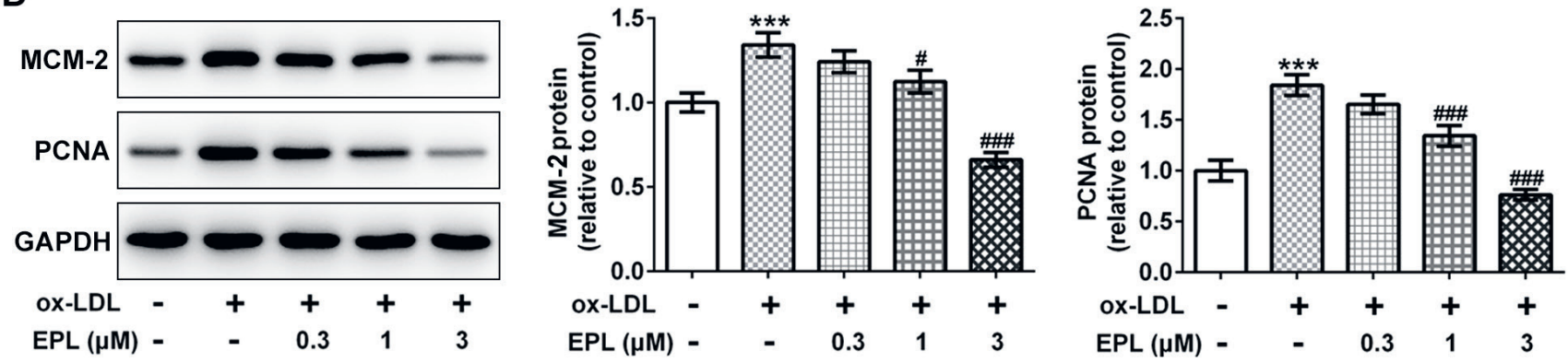

Fig. 1. EPL dose-dependently inhibited cell proliferation in ox-LDL-stimulated VSMCS

A. Cell viability was detected using a CCK-8 assay when VSMCs were treated with different concentrations of EPL; B. CCK-8 assay was employed to determine viability of VSMCs in the presence or absence of ox-LDL and EPL; C. The expression of Ki67 was measured using immunofluorescence staining (magnification $\times 200)$; D. Western blot analysis was used to analyze the levels of MCM-2 and PCNA. All experiments were repeated 3 times independently $(n=3)$.** $p<0.01$, ${ }^{* * *} p<0.001$ compared to control; ${ }^{*} \mathrm{p}<0.05$, $\# \mathrm{p}<0.01$ and ${ }^{\# \# \#} \mathrm{p}<0.001$ compared to ox-LDL; EPL - eplerenone; Ox-LDL - oxidized low-density lipoprotein; VSMCs - vascular smooth muscle cells; CCK-8 - cell counting kit-8; MCM-2 - minichromosome maintenance-2; PCNA - proliferating cell nuclear antigen.

remarkably promoted the migration of VSMCs relative to the control group. Conversely, EPL dose-dependently declined the ox-LDL-promoted ability of cell migration. As expected, results of transwell migration assay presented the same trends with those of the scratch wound healing assay (Fig. 2C,D). Simultaneously, EPL dramatically downregulated the expression of migration-associated proteins including MMP2 and MMP9 in a dose-dependent manner (Fig. 2E). Overall, these data suggest that EPL treatment represses migration of VSMCs boosted by ox-LDL.

\section{EPL downregulated the expression of GPER in VSMCs exposed to ox-LDL}

To uncover the potential mechanisms of EPL in VSMCs under ox-LDL exposure, the STITCH website (http://stitch. embl.de) was applied to search the potential proteins interacting with EPL. The GPER was assumed to combine with EPL (Fig. 3A). It is observable in Fig. 3B that GPER expression was gradually enhanced with the increased concentrations of ox-LDL. Moreover, under exposure to $100 \mu \mathrm{g} / \mathrm{mL}$ of ox-LDL at time points from $12 \mathrm{~h}$ to $72 \mathrm{~h}$, the level of GPER in VSMCs was markedly upregulated compared with the control group, and the highest level of GPER was noted at $48 \mathrm{~h}$ (Fig. 3C). Afterwards, the effect of EPL on GPER expression was assessed using western blot analysis. Figure 3D displays that EPL dose-dependently lowered the level of GPER induced by ox-LDL. To sum up, these observations reveal that EPL can inhibit GPER expression in VSMCs exposed to ox-LDL.

\section{GPER overexpression reversed the inhibitory impacts of EPL exposure on proliferation and migration of VSMCs treated with ox-LDL}

Subsequently, to study the exact regulatory mechanisms of EPL on GPER, GPER was overexpressed by transfection with overexpression plasmids. Successful transfection was presented in Fig. 4A, and VSMCs transfected with Oe-GPER-1 were selected for the following experiments due to Oe-GPER-1 producing higher expression of GPER. 
A
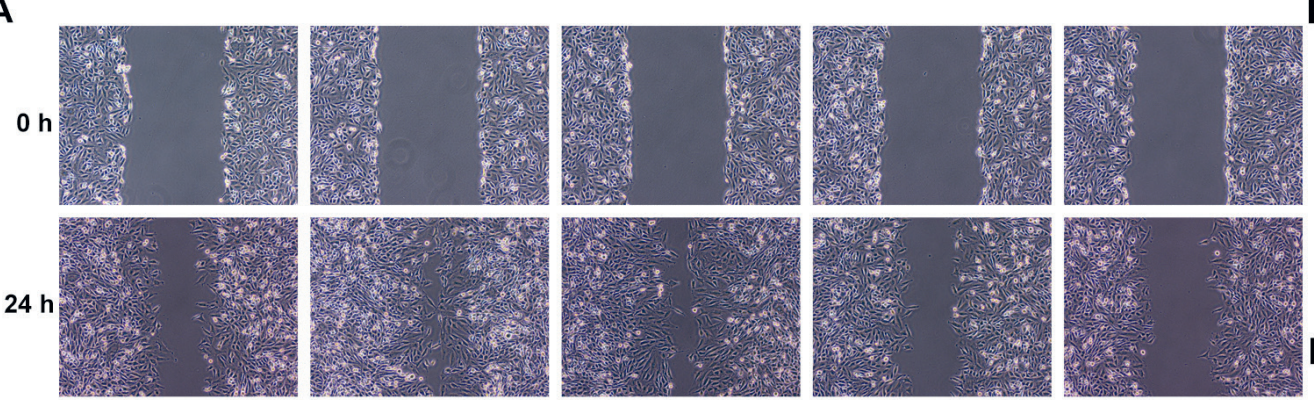

B
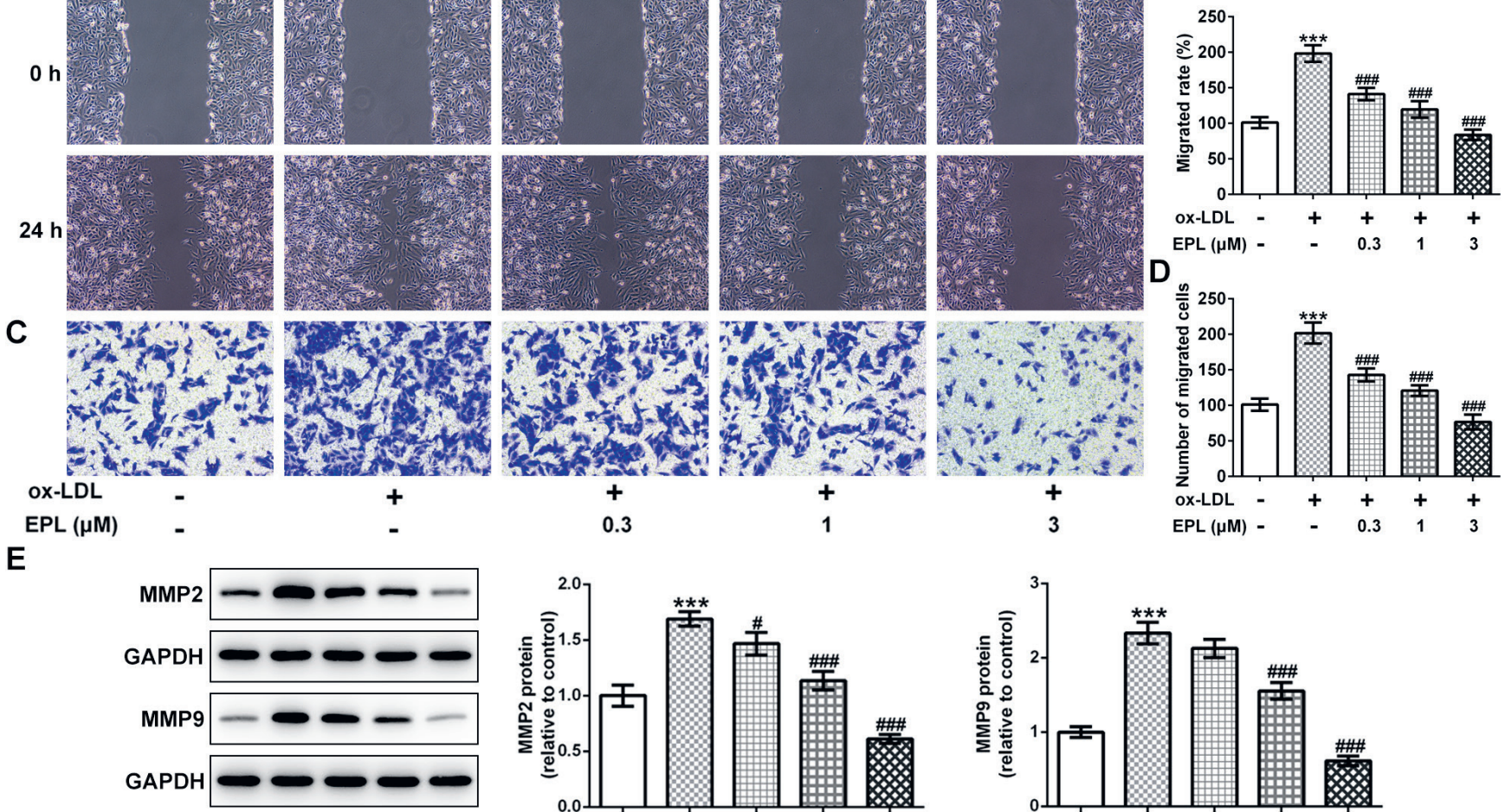

ox-LDL $\quad-\quad+\quad+\quad+\quad+$
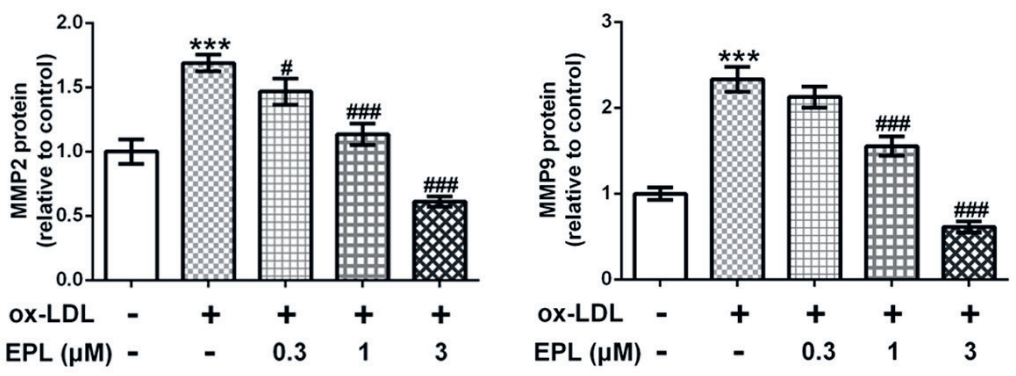

Fig. 2. EPL treatment suppressed migration of VSMCs induced by ox-LDL

A and B. Cell migration was determined using scratch wound healing assay (magnification $\times 100$ ); C and D. Cell migration was assessed using transwell migration assay (magnification $\times 200$ ): E. The expression of MMP2 and MMP9 was examined using western blot analysis. The experiments were generated from 3 independent repeats $(n=3)$. ${ }^{* *} p<0.001$ compared to control; ${ }^{*} p<0.05$ and ${ }^{\# \# \# ~} p<0.001$ compared to ox-LDL. EPL - eplerenone; ox-LDL - oxidized low-density lipoprotein; VSMCs - vascular smooth muscle cells; MMP - matrix metalloproteinase.

A

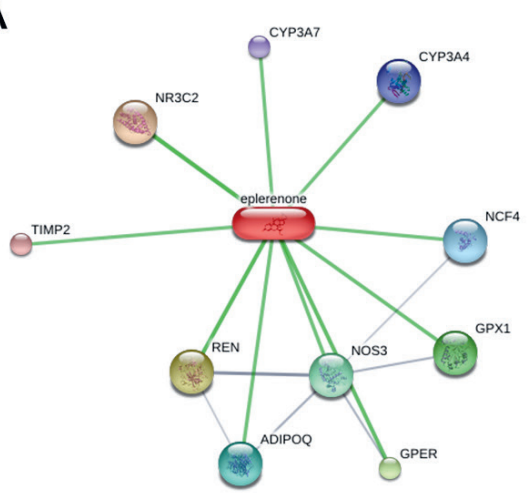

C
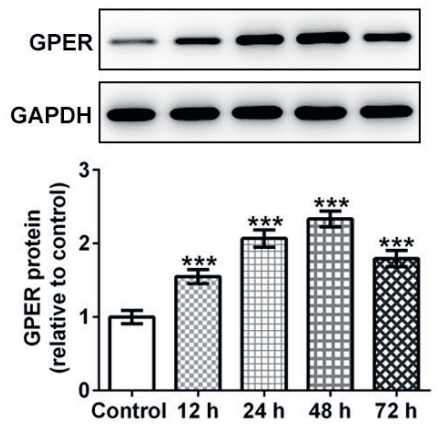

B
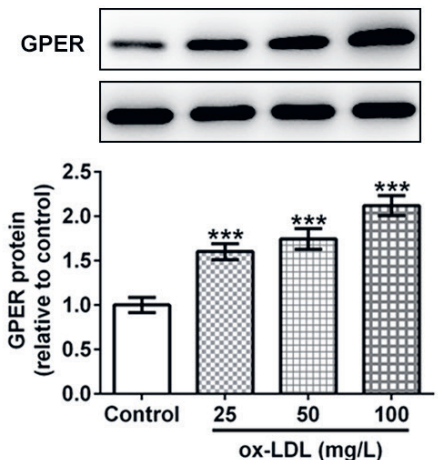

D
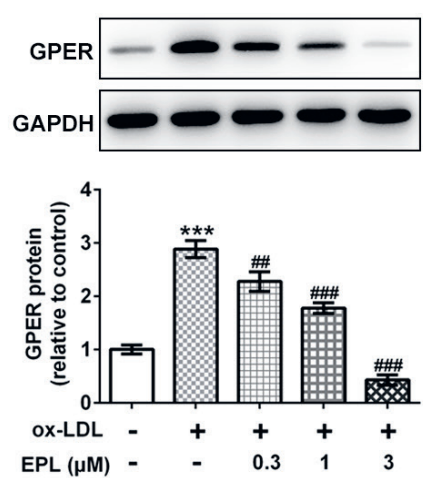

Fig. 3. EPL decreased GPER expression in ox-LDLtreated VSMCs

A. The EPL-protein interaction network was predicted using the STITCH website; B. Western blot analysis of the expression of GPER when VSMCS were exposed to $25 \mathrm{mg} / \mathrm{L}, 50 \mathrm{mg} / \mathrm{L}$ and $100 \mathrm{mg} / \mathrm{L}$ of ox-LDL, respectively; $C$. The level of GPER was examined using western blot analysis in VSMCs stimulated by $100 \mathrm{mg} / \mathrm{L}$ of ox-LDL for $12 \mathrm{~h}, 24 \mathrm{~h}$ $48 \mathrm{~h}$, and $72 \mathrm{~h}$, respectively. The experiments were generated from 3 independent repeats $(n=3)$; D. GPER expression was measured using western blot analysis when VSMCs were treated with a series of concentrations of EPL. All experiments were repeated 3 times independently $(n=3) .{ }^{* * *} p<0.001$ compared to control; $\# p<0.01$ and $\# \# p<0.001$ compared to ox-LDL; EPL - eplerenone; ox-LDL - oxidized low-density lipoprotein; VSMCs - vascular smooth muscle cells; GPER - G protein-coupled estrogen receptor. 
A

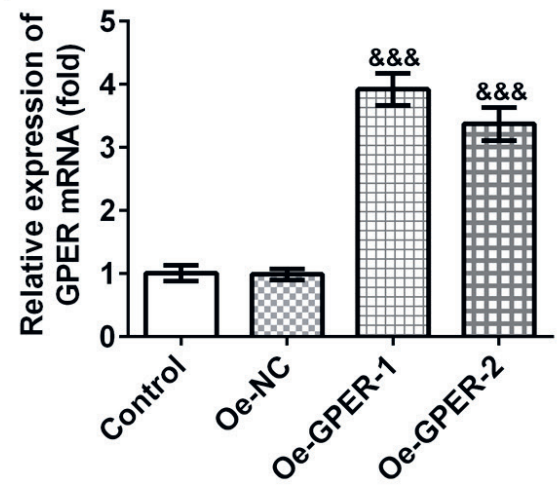

D

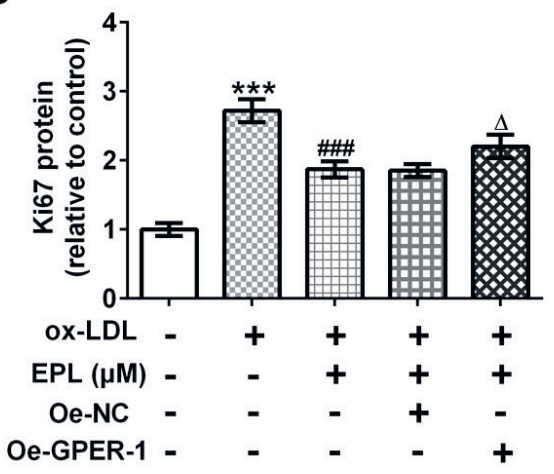

B
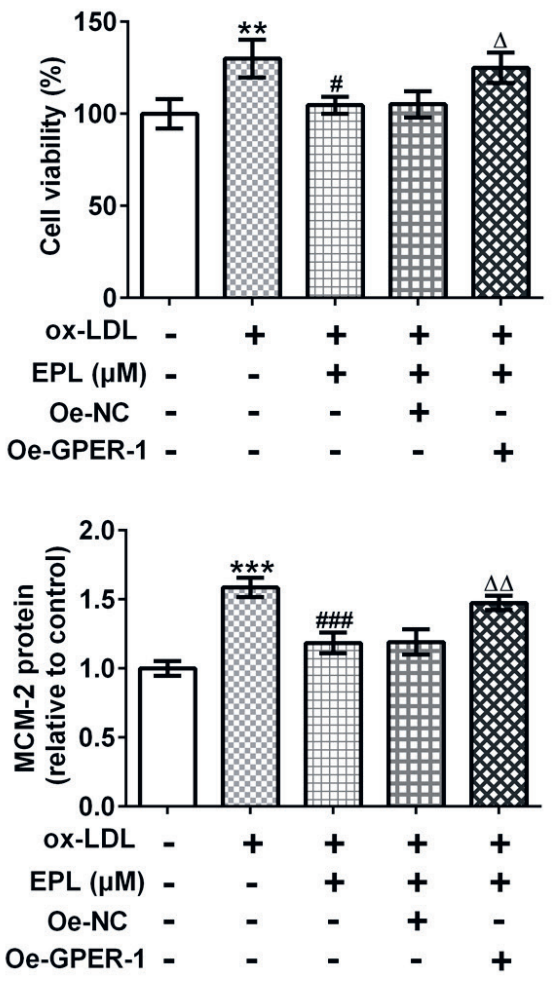

C

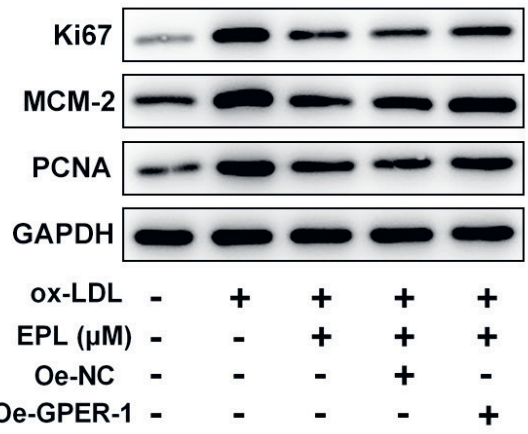

Fig. 4. GPER overexpression alleviated the inhibitory effect of EPL exposure on proliferation of VSMCs induced by ox-LDL

A. The expression of GPER was determined using RT-qPCR after transfection with Oe-GPER-1 or Oe-GPER-2. The experiments were generated from 3 independent repeats $(n=3)$. \&\&\& $p<0.001$ compared to Oe-NC; B. Cell viability was evaluated using a CCK-8 Kit; C and D. Western blot analysis was employed to assess the expression of proliferation-related proteins. The experiments were generated from 3 independent repeats $(n=3$ ).

${ }^{* *} \mathrm{p}<0.01$ and ${ }^{* * *} \mathrm{p}<0.001$ compared to control; $\mathrm{*} p<0.05$ and ${ }^{\# \# \#} \mathrm{p}<0.001$ compared to ox-LDL; ${ }^{\Delta} \mathrm{p}<0.05$ and ${ }^{\Delta \Delta} \mathrm{p}<0.01$ compared to ox-LDL + EPL + Oe-NC; EPL - eplerenone; ox-LDL - oxidized low-density lipoprotein; Oe-NC - empty vector; VSMCs - vascular smooth muscle cells; GPER - G proteincoupled estrogen receptor; CCK-8 - cell counting kit-8; MCM-2 - minichromosome maintenance-2; PCNA - proliferating cell nuclear antigen.

As shown in Fig. 4B, GPER overexpression significantly elevated cell viability relative to the Oe-NC group. Meanwhile, as compared to VSMCs transfected with Oe-NC, the decrease of Ki67, MCM-2 and PCNA was notably reversed after GPER overexpression (Fig. 4C,D). Results presented in Fig. 5A-D revealed that the ability of cell migration was remarkably enhanced in the GPER overexpression group compared with the empty vector group. Moreover, GPER-upregulation conspicuously increased the levels of MMP2 and MMP9. Through the above findings, we prove that GPER overexpression abolished the suppressive effects of EPL exposure on proliferation and migration of VSMCs treated by ox-LDL.

\section{Discussion}

It is well known that AS is a chronic degenerative disease and has become a major cause of cardiovascular morbidity and mortality. ${ }^{15}$ Aging, obesity, diabetes mellitus, chronic inflammation, and elevated plasma ox-LDL are risk factors for the disease process. ${ }^{16,17}$ Moreover, postoperative care, rehabilitation training and health education after AS bypass grafting is important in improving the quality of life of patients and preventing the occurrence of poor prognostic event. Existing studies have demonstrated that EPL possesses protective functions against cardiovascular disease. ${ }^{18}$ The present study was the first to explore the roles of EPL in the functions of VSMCs as applied to AS.

The VSMCs are the major cell type observed in blood vessel walls that play considerable roles in the regulation of multiple physiological and pathological situations. ${ }^{19}$ Aberrant proliferation and migration of VSMCs are the key events in the progression of AS and restenosis after percutaneous coronary intervention. ${ }^{20}$ An increasing number of studies have reported that ox-LDL exerts a promotion effect in the development of AS by stimulating the proliferation of VSMCs within the vessel wall. ${ }^{21,22}$ Therefore, agents blocking or inhibiting proliferation and migration of VSMCs induced by ox-LDL may contribute to the identification of therapeutic strategies. Eplerenone is a newly found novel selective aldosterone receptor antagonist. ${ }^{8,9}$ Additionally, it has been reported to alleviate hepatocellular carcinoma growth and angiogenesis in mice. ${ }^{23}$ Adriamycin nephropathy rats treated with EPL exhibit obvious 
A

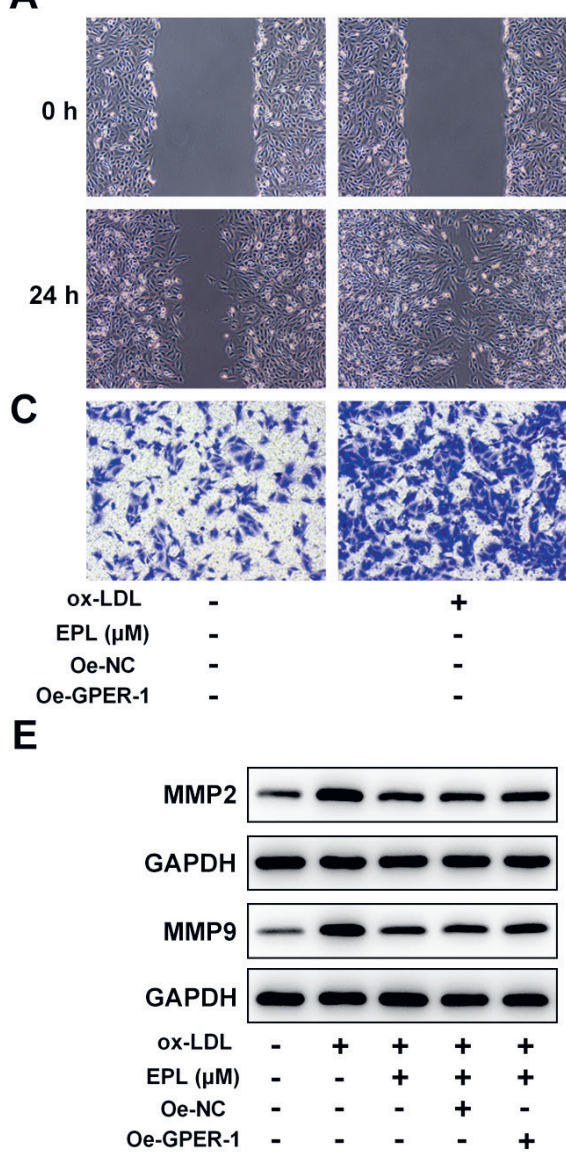

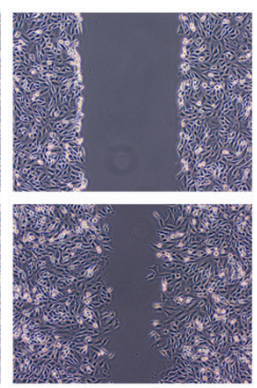
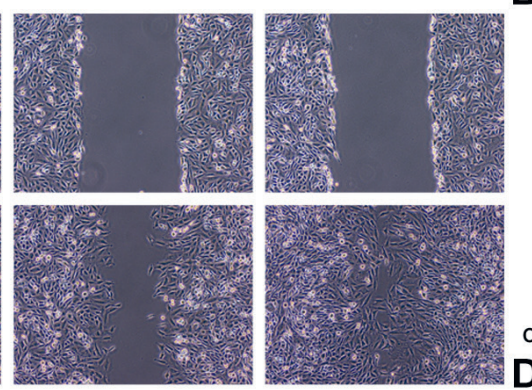

B
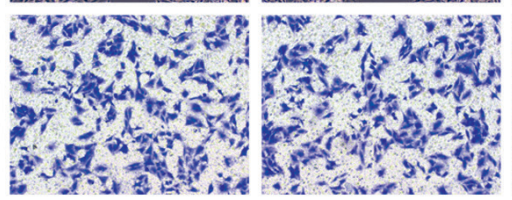

$+$

$+$
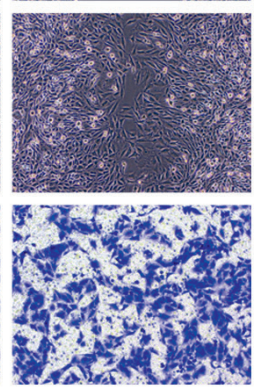

$+$

$+$

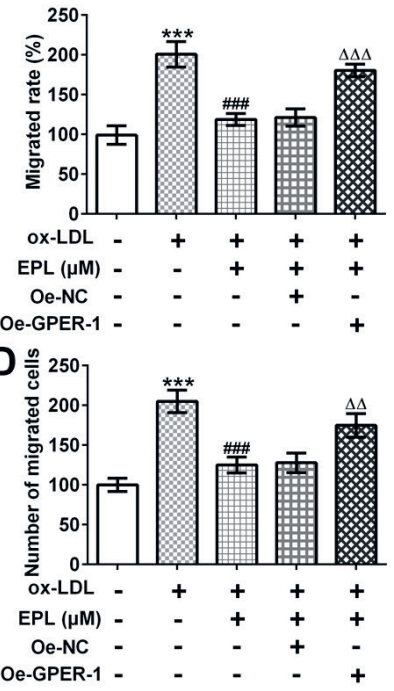

Fig. 5. GPER overexpression attenuated the inhibitory effect of EPL treatment on migration of VSMCs induced with ox-LDL

A and B. Cell migration was determined using scratch wound healing assay (magnification $\times 200$ ); C and D. Cell migration was assessed using transwell migration assay (magnification $\times 200$ ); E. The expression of MMP2 and MMP9 was examined using western blot analysis. The experiments were generated from 3 independent repeats $(n=3)$. ${ }^{* *} p<0.001$ compared to control; $\# p<0.05$ and ${ }^{\# \# \# p ~} 0.001$ compared to ox-LDL;

Oe-NC - empty vector; EPL - eplerenone; Ox-LDL - oxidized low-density lipoprotein; VSMCs - vascular smooth muscle cells; GPER - G protein-coupled estrogen receptor; MMP - matrix metalloproteinase.

attenuation of mesangial cell proliferation and matrix expansion..$^{24}$ Moreover, EPL can inhibit neointimal formation after coronary stent implantation in swine by decreasing collagen accumulation. ${ }^{25}$ The present study suggested that EPL treatment significantly hampered proliferation and migration of VSMCs treated with ox-LDL, suggesting a promising therapeutic agent in the treatment of AS.

We further explored the molecular mechanisms underlying the inhibitory roles of EPL on ox-LDL-stimulated VSMCs. The STITCH website (http://stitch.embl.de) was used to detect the potential proteins that could partake in the regulation of EPL. Thereafter, GPER, a G proteincoupled receptor coupled with Gs proteins, was discovered as one binding point of EPL. According to published studies, GPER promotes cell proliferation and migration of triple-negative breast cancer, renal cell carcinoma and ovarian cancer. ${ }^{26-28}$ Furthermore, activation of the GPER can increase neurogenesis and alleviate neuroinflammation in the hippocampus of male spontaneously hypertensive rats. ${ }^{29}$ Also, blocking of GPER function mitigates the bisphenol A-stimulated proliferation of VSMCs. ${ }^{14}$
Results of the current work indicated that GPER expression was markedly upregulated in ox-LDL-induced VSMCs and the elevation was abolished under treatment with EPL. Importantly, GPER overexpression dramatically reversed the inhibitory effects of EPL intervention on proliferation and migration of VSMCs stimulated by ox-LDL, which was accompanied by the expression changes of proliferationand migration-associated proteins.

\section{Conclusions}

To the best of our knowledge, the present study was the first to investigate the pivotal roles of EPL on VSMCs function. These findings demonstrate that EPL restricts proliferation and migration of VSMCs stimulated by oxLDL partly by downregulating GPER expression, providing experimental and mechanistic support for EPL use in the clinical treatment of AS. However, a lack of the study in vivo is a limitation of the present research, and therefore, a comprehensive analysis is required in the future. 


\section{ORCID iDs}

Dandan Wang (D) https://orcid.org/0000-0003-0808-5419 Minglei Wang (1) https://orcid.org/0000-0002-6819-0571 Pingping Sun (D) https://orcid.org/0000-0001-9954-0303 Qiaoyan Gao (D) https://orcid.org/0000-0002-7941-3099

\section{References}

1. Zhang B, Dong Y, Zhao Z. LncRNA MEG8 regulates vascular smooth muscle cell proliferation, migration and apoptosis by targeting PPARalpha. Biochem Biophys Res Commun. 2019;510(1):171-176. doi:10. 1016/j.bbrc.2019.01.074

2. Luscher TF. Atherosclerosis and CAD. Eur Heart J. 2015;36(8):457-459. doi:10.1093/eurheartj/ehv002

3. Torres N, Guevara-Cruz M, Velazquez-Villegas LA, Tovar AR. Nutrition and atherosclerosis. Arch Med Res. 2015;46(5):408-426. doi:10.1016/j. arcmed.2015.05.010

4. Chistiakov DA, Orekhov AN, Bobryshev YV. Vascular smooth muscle cell in atherosclerosis. Acta Physiol (Oxf). 2015;214(1):33-50. doi:10. 1111/apha.12466

5. Chen Z, Pan X, Sheng Z, Yan G, Chen L, Ma G. Baicalin suppresses the proliferation and migration of Ox-LDL-VSMCs in atherosclerosis through upregulating miR-126-5p. Biol Pharm Bull. 2019;42(9): 1517-1523. doi:10.1248/bpb.b19-00196

6. Zheng J, Chen K, Wang H, et al. SIRT7 regulates the vascular smooth muscle cells proliferation and migration via Wnt/beta-catenin signaling pathway [erratum published in:Biomed Res Int. 2019;2019:8014381. doi:10.1155/2019/8014381]. Biomed Res Int. 2018;2018:4769596. doi:10. $1155 / 2018 / 4769596$

7. Wang B, Ge Z, Cheng Z, Zhao Z. Tanshinone IIA suppresses the progression of atherosclerosis by inhibiting the apoptosis of vascular smooth muscle cells and the proliferation and migration of macrophages induced by ox-LDL. BiolOpen. 2017;6(4):489-495. doi:10.1242/ bio. 024133

8. Craft J. Eplerenone (Inspra), a new aldosterone antagonist for the treatment of systemic hypertension and heart failure. Proc (Bayl Univ Med Cent). 2004;17(2):217-220. doi:10.1080/08998280.2004.11927973

9. Dizaye K, Mustafa ZA. The effect of eplerenone on the renin-angiotensin-aldosterone system of rats with thyroid dysfunction.J Pharm Pharmacol. 2019;71(12):1800-1808. doi:10.1111/jphp.13168

10. Shao PP, Liu CJ, Xu Q, et al. Eplerenone reverses cardiac fibrosis via the suppression of Tregs by inhibition of Kv1.3 channel. Front Physiol. 2018;9:899. doi:10.3389/fphys.2018.00899

11. Wang $\mathrm{CH}$, Wang $\mathrm{Z}$, Liang LJ, et al. The inhibitory effect of eplerenone on cell proliferation in the contralateral kidneys of rats with unilateral ureteral obstruction. Nephron. 2017;136(4):328-338. doi:10. $1159 / 000473702$

12. Chen H, Sun F, Zhong X, Shao Y, Yoshimura A, Liu Y. Eplerenonemediated aldosterone blockade prevents renal fibrosis by reducing renal inflammation, interstitial cell proliferation and oxidative stress. Kidney Blood Press Res. 2013;37(6):557-566. doi:10.1159/000355736

13. Yang K, Yao Y. Mechanism of GPER promoting proliferation, migration and invasion of triple-negative breast cancer cells through CAF. Am J Trans/ Res. 2019;11(9):5858-5868. PMID:31632554

14. Gao F, Huang Y, Zhang L, Liu W. Involvement of estrogen receptor and GPER in bisphenol A induced proliferation of vascular smooth muscle cells. Toxicol In Vitro. 2019;56:156-162. doi:10.1016/j.tiv.2019.01.012
15. Yu MH, Lin MC, Huang CN, Chan KC, Wang CJ. Acarbose inhibits the proliferation and migration of vascular smooth muscle cells via targeting Ras signaling. Vascul Pharmacol. 2018;103-105:8-15. doi:10. 1016/j.vph.2018.02.001

16. Anogeianaki A, Angelucci D, Cianchetti E, et al. Atherosclerosis: A classic inflammatory disease. Int J Immunopathol Pharmacol. 2011;24(4): 817-825. https://doi.org/10.1177/039463201102400401

17. Huang $Y L, H u Y Z$, Mai WY, et al. Plasma oxidized low-density lipoprotein is an independent risk factor in young patients with coronary artery disease. Dis Markers. 2011;31(5):295-301. doi:10.3233/DMA2011-0832

18. Stewart Coats AJ, Shewan L. Eplerenone's role in the management of complex cardiovascular disorders. Int J Cardiol. 2015;200:1-2. https://doi.org/10.1016/j.ijcard.2015.05.128

19. Eun SY, Ko YS, Park SW, Chang KC, Kim HJ. IL-1beta enhances vascular smooth muscle cell proliferation and migration via $\mathrm{P} 2 \mathrm{Y} 2$ receptormediated RAGE expression and HMGB1 release. Vascul Pharmacol. 2015;72:108-117. doi:10.1016/j.vph.2015.04.013

20. Fan TF, He JH, Yin YQ, et al. Dioscin inhibits intimal hyperplasia in rat carotid artery balloon injury model through inhibition of the MAPKFoxM1 pathway. Eur J Pharmacol. 2019;854:213-223. doi:10.1016/j. ejphar.2019.03.050

21. Pirillo A, Norata GD, Catapano AL. LOX-1, OxLDL, and atherosclerosis. Mediators Inflamm. 2013;2013:152786. doi:10.1155/2013/152786

22. Yang N, Dong B, Song YQ, et al. Downregulation of miR- 637 promotes vascular smooth muscle cell proliferation and migration via regulation of insulin-like growth factor-2. Cell Mol Biol Lett. 2020;25(1):12. doi:10.1186/s11658-020-00222-z

23. Kaji K, Yoshiji H, Kitade M, et al. Selective aldosterone blocker, eplerenone, attenuates hepatocellular carcinoma growth and angiogenesis in mice. Hepatol Res. 2010;40(5):540-549. doi:10.1111/j.1872-034X. 2010.00636.x

24. Fang Z, Zhang C, He FF, et al. Protective effects of eplerenone on podocyte injury in adriamycin nephropathy rats. J Huazhong Univ Sci Technolog Med Sci. 2011;31(3):329-334. doi:10.1007/s11596-011-0376-5

25. Wakabayashi K, Suzuki H, Sato T, Iso Y, Katagiri T, Takeyama Y. Eplerenone suppresses neointimal formation after coronary stent implantation in swine. Int J Cardiol. 2006;107(2):260-266. doi:10.1016/j.ijcard. 2005.03.078

26. Guan BZ, Yan RL, Huang JW, et al. Activation of G protein coupled estrogen receptor (GPER) promotes the migration of renal cell carcinoma via the PI3K/AKT/MMP-9 signals. Cell Adhes Migr. 2018;12(2): 109-117. doi:10.4161/19336918.2014.990781

27. Wang Y, Peng ZQ, Meng R, et al. NHERF1 inhibits proliferation of triplenegative breast cancer cells by suppressing GPER signaling. Oncol Rep. 2017;38(1):221-228. doi:10.3892/or.2017.5649

28. Yan Y, Jiang XL, Zhao Y, Wen HX, Liu GY. Role of GPER on proliferation, migration and invasion in ligand-independent manner in human ovarian cancer cell line SKOV3. Cell Biochem Funct. 2015;33(8):552-559. doi:10.1002/cbf.3154

29. Correa J, Ronchetti S, Labombarda F, De Nicola AF, Pietranera L. Activation of the $G$ protein-coupled estrogen receptor (GPER) increases neurogenesis and ameliorates neuroinflammation in the hippocampus of male spontaneously hypertensive rats. Cell Mol Neurobiol. 2020;40(5):711-723. doi:10.1007/s10571-019-00766-5 